\title{
Increased expression of serine biosynthetic pathway genes is associated with skeletal muscle hypertrophy in sheep
}

\author{
T. Parr, S. Al-Doski, K. Hemmings, Z. Daniel, D. Brown, C. Lu, C. Hodgman, S. May and \\ J. Brameld \\ School of Biosciences, University of Nottingham, Sutton Bonington Campus, Loughborough, LE12 5RD
}

Beta-adrenergic agonists (BA) and Growth Hormone (GH) induce muscle hypertrophy, associated with changes in nutrient mobilisation and utilization ${ }^{(1)}$. The aim of this present study was to investigate the mechanisms that mediate these effects in sheep treated with BA or GH for 6 days, by examining the changes in skeletal muscle transcriptome and blood metabolome.

Male lambs (120 days old) were all fed a high protein/energy diet ad-libitum, with the GH group $(\mathrm{n}=10)$ receiving a single subcutaneous injection of bovine $\mathrm{GH}(3.75 \mathrm{mg} / \mathrm{kg}$ body weight, POSILAC, Monsanto) on day 1; the BA group ( $\mathrm{n}=10)$ receiving BA (cimaterol) at $10 \mathrm{mg} / \mathrm{kg}$ feed, whereas the control group $(\mathrm{CO}, \mathrm{n}=11)$ only had the ad-libitum feed. After 6 days sheep were slaughtered, plasma and samples of the Longissimus dorsi (LD), Supraspinatus (SS) muscles and liver were collected. The relative mRNA expression of the adult myosin heavy chain isoforms (MyHC I, IIA, IIX and IIB) were determined ${ }^{(2)}$. Metabolomic analysis of plasma samples was carried out by Metabolon Inc (USA) using GC/MS and LC/MS/MS platforms. The effect of treatments on the LD transcriptome was assessed on a subset of samples ( $\mathrm{n}=3$ from each treatment) via a cross-species approach ${ }^{(3)}$ using the Affymetrix Human $\mathrm{U} 133+2$ GeneChip array (47 K human microarray). Verification of identified genes and proteins was by quantitative RT-PCR or western blotting, respectively, on LD, SS and livers from all animals.

BA, but not $\mathrm{GH}$, significantly $(\mathrm{P}<0.05)$ increased muscle weights and this was associated with an increase in MyHCIIX/IIB mRNA and decrease in MyHCIIA mRNA expression $(\mathrm{P}<0.05)$, indicating a transition to large fast-glycolytic muscle fibre types. Products of triacylglycerol breakdown, including glycerol, monoacylglycerol and free fatty acids were all elevated in the plasma of both BA and GH treatments, but the free fatty acid profile suggested the effects were more pronounced with GH treatment $(\mathrm{P}<0.05)$. Likewise $\mathrm{GH}$ rather than $\mathrm{BA}$ had a greater impact on elevating plasma glucose and associated metabolites $(\mathrm{P}<0.05)$, indicative of insulin resistance. Analysis of the LD transcriptome identified 477 and 316 transcripts were significantly altered $(\mathrm{P}<0.05$ and 1.5 fold change) by BA and GH respectively, relative to controls. Gene ontogeny analysis (Genespring) of these transcripts indicated more pathways affected by BA than GH. BA decreased in oxidative respiration pathways (TCA cycle and oxidative phosphorylation) and upregulated serine biosynthetic pathway. Subsequent qRT-PCR analysis showed increases in expression of phosphoglycerate dehydrogenase $(\mathrm{PHGDH})((\mathrm{P}<0.001)$ and phosphoserine-aminotransferase $(\mathrm{PSAT})(\mathrm{P}<0.001) \mathrm{mRNA}$, along with an increase $(\mathrm{P}<0.01)$ in PHGDH protein in muscle from BA treated sheep relative to both control and GH treated sheep. Metabolomic analysis of plasma amino acids indicated no effect of BA treatment on plasma serine concentrations compared to controls, but a significant decrease in $\mathrm{GH}$ treated sheep $(\mathrm{P}<0 \cdot 05)$.

The serine biosynthetic pathway has been shown to be upregulated in various cancers and is thought to be a novel mechanism for hyperplastic growth ${ }^{(4)}$. We show for the first time that upregulation of the serine biosynthetic pathway is also associated with BA stimulated muscle hypertrophic growth. BA had a stronger muscle hypertrophic effect than $\mathrm{GH}$ over this short timeframe. Unlike GH, BA does not appear to have a major effect upon the systemic mobilisation of nutrients, but instead seems to targets muscle, activating muscle biosynthetic pathways that potentially provide the substrates required for growth.

This work was supported by the Biotechnology and Biological Sciences Research Council (BBSRC).

1. Bell AW, Bauman DE, Beermann DH et al. (1998) J Nutr 128, 360S-363S.

2. Hemmings K, Parr T, Daniel ZC et al. (2009) J Anim Sci 87, 3915-22.

3. Graham NS, May ST, Daniel ZC et al. (2011) Animal 5, 861-6.

4. Amelio I, Cutruzzola F, Antonov A et al. (2014) Trends Biochem Sci 39, 191-198. 